\title{
Small-scale Structure via Flows
}

\author{
Albert M. Fisher
}

\begin{abstract}
We study the small scale of geometric objects embedded in a Euclidean space by means of the flow defined by zooming toward a point in the object. For Fuchsian and Kleinian limit sets this "scenery flow" corresponds naturally to the geodesic flow. We explore this analogy in a variety of examples, coming from dynamics, geometry and probability theory.
\end{abstract}

Mathematics Subject Classification (2000). 37D40, 37F50, 60J65, 60G18, 37A40, 37A50, 60F17.

Keywords. Geodesic flow, horocycle flow, scenery flow, Brownian local time, Kleinian limit set, Julia set, scaling function, Hausdorff dimension, average density.

\section{Introduction}

Suppose we center a mathematical microscope at some point of a fractal set, and turn the knob continuously; as we zoom down toward smaller scales, ever-changing scenes go past us, as if we were riding on a train taking us deeper and deeper into the heart of the fractal landscape. Let us try to model such a fractal excursion mathematically. The continuously changing nature of the process suggests that a precise description will involve a continuous-time dynamical system, in other words a flow. In this article, we shall sketch how such a flow (the scenery flow of the fractal set) can be defined, and indeed, constructed rigorously for a variety of examples, and we shall see how the scenery flow can be usefully applied in studying the fractal geometry. We shall, moreover, see that this flow of magnification, and a related translation flow, provide close analogues of two familiar flows: the geodesic and horocycle flows of a Riemann surface.

To begin our story, we shall recall some basic properties of these classical flows. 


\section{Geodesic and horocycle flows}

In the complex plane $\mathbb{C}$, let $\mathbb{H}=\{z=x+i y: y \geq 0\}$ denote the upper half plane. The interior of $\mathbb{H}$, those points with $y>0$, is given the hyperbolic metric, defined by $d s^{2}=\left(d x^{2}+d y^{2}\right) / y^{2}$, which makes it isometric to the Poincaré disk $\Delta$. The orientation-preserving isometries for this metric are the real Möbius transformations $\operatorname{Möb}(\mathbb{R})$, with

$$
f_{A}(z)=(a z+b) /(c z+d)
$$

for $A=\left[\begin{array}{ll}a & b \\ c & d\end{array}\right]$ where $a, b, c, d \in \mathbb{R}$ and $\operatorname{det}(A)=a d-b c=1$; that is, $A \in S L(2, \mathbb{R})$, the special linear group.

The matrices $A$ and $\gamma A$ for $\gamma \neq 0$ give the same Möbius transformation, and this is the only such identification, so $\operatorname{Mob}(\mathbb{R}) \cong P S L(2, \mathbb{R})$, the projective special linear group. Let $\Gamma \subseteq \operatorname{Möb}(\mathbb{R})$ be a discrete subgroup. Then the identification space $\Gamma \backslash \mathbb{H}$ is a Riemann surface; this may be compact, or be noncompact with either finite or infinite area. The unit tangent bundle of $\mathbb{H}$ can be identified with $P S L(2, \mathbb{R})$. This correspondence is easily described. We will associate (arbitrarily) the identity matrix $I$ to the vector $i_{i}$ which is located at the point $i \in \mathbb{H}$ and points in the vertical direction; then, given $A \in S L(2, \mathbb{R})$, let $f_{A}^{*}\left(i_{i}\right)$ be the image of this vector by the derivative map of $f_{A}$, that is, it is the vector located at the point $f_{A}(i)$ which has been rotated appropriately by the argument of the complex derivative. This image vector also has hyperbolic length one, as Möbius transformations are isometries for the hyperbolic metric; so this defines a map from $P S L(2, \mathbb{R})$ to the unit tangent bundle $T_{1}(\mathbb{H})$. The group $\Gamma$ acts on $P S L(2, \mathbb{R})$ by left multiplication and one sees that $\Gamma \backslash P S L(2, \mathbb{R})$ is the unit tangent bundle of the surface $\Gamma \backslash \mathbb{H}$.

The geodesic flow on the surface is by definition the flow on this unit tangent bundle which moves a vector along its tangent geodesic at unit speed. Algebraically, this is given by right multiplication by $E_{t} \equiv\left[\begin{array}{cc}e^{\frac{t}{2}} & 0 \\ 0 & e^{-\frac{t}{2}}\end{array}\right]$ on $\Gamma \backslash P S L(2, \mathbb{R})$. To understand this, note that this matrix is equivalent as a Möbius transformation to $\left[\begin{array}{ll}e^{t} & 0 \\ 0 & 1\end{array}\right]$ which dilates the plane by the factor $e^{t}$, and hence moves the vector $i_{i}$ up the imaginary axis at unit speed in the hyperbolic metric. The action on a general unit vector is then given by the conjugation by $f_{A}^{*}$ which is a hyperbolic isometry, so this is indeed the geodesic flow. The unstable horocycle flow $h_{t}^{u}$ is given by the right action of $H_{t}^{u} \equiv\left[\begin{array}{ll}1 & 0 \\ t & 1\end{array}\right]$; the stable flow acts by its transpose. As the names suggest, these preserve the unstable and stable horocycles. Recall here that the stable set of a point $x$ in a flow $\tau_{t}$ is the set of all $y$ which are forward asymptotic to $x$, i.e. such that $d\left(\tau_{t}(x), \tau_{t}(y)\right) \rightarrow 0$ as $t \rightarrow+\infty$, while for the unstable set we trade + for $-\infty$. The stable set of the point $i_{i}$ for the geodesic flow is the set of all vertical unit vectors based on the line $y=1$, see the right side of Fig. 2 . This line is a "circle" tangent to $\infty$. Therefore by conjugation, stable sets of the geodesic 

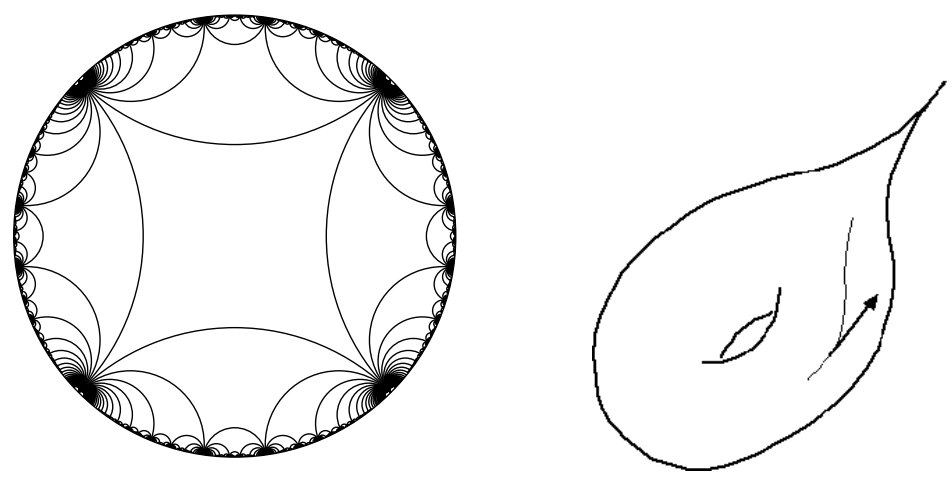

Figure 1. Covering space for punctured torus; the geodesic flow

flow are one dimensional, and are exactly the orbits of the stable horocycle flow, which are carried by those circles classically known as horocycles: those tangent to the boundary $\mathbb{R}$ of $\mathbb{H}$.

For the simplest example of a noncompact, finite area surface, see Fig.1; here (depicted in the disk model) $\Gamma$ is a free group on two generators, these being two hyperbolic Möbius transformations, one which shoves the interior of the disk to the right and one which moves everything up; the curved quadrilateral in the center is a fundamental domain for this action. The left side is glued to the right, and the bottom to the top, so the resulting surface is a torus, just like for the usual gluings of a square, to get the quotient space $\mathbb{R}^{2} / \mathbb{Z}^{2}$, except that now the corner point gives a cusp, as it goes out to $\infty$ in the hyperbolic metric: this is a punctured torus (Fig. 1). Classical results are:

Theorem 2.1. The geodesic and horocycle flows $g_{t}, h_{t}^{u}, h_{t}^{s}$ preserve Riemannian volume of the unit tangent bundle of the surface $M$. This measure is finite iff the surface area is finite. For this case, if $M$ is compact (equivalently has no cusps) then:

(i) $g_{t}$ is ergodic, indeed is measure-theoretically isomorphic to a Bernoulli flow of finite entropy $(=1)$;

(ii) $h_{t}^{u}, h_{t}^{s}$ are uniquely ergodic, with entropy zero.

In the finitely generated, finite area case with cusps, all this is true except that $h_{t}^{u}, h_{t}^{s}$ are only nearly uniquely ergodic; normalized Riemannian volume is the only non-atomic invariant probability measure if we disallow measures which give mass to horocycle orbits tangent to cusps.

More interesting for us will be the infinite area case, where the cusp opens up to flare out in a hyperbolic trumpet, Fig. 3, we return to this below. 

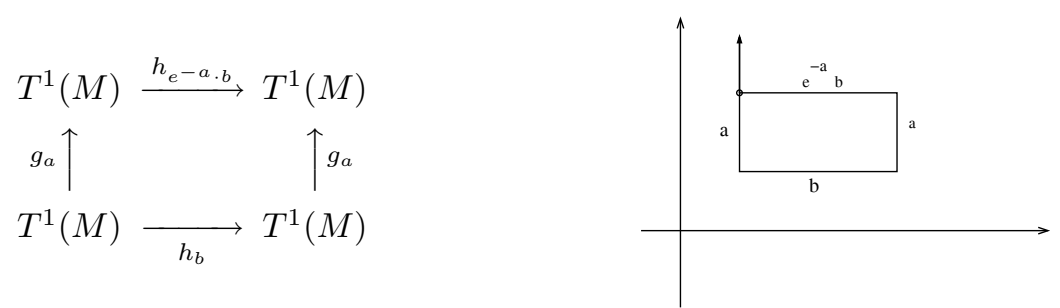

Figure 2. A commutative diagram, seen in the upper half plane; hyperbolic distances $=$ flow times.
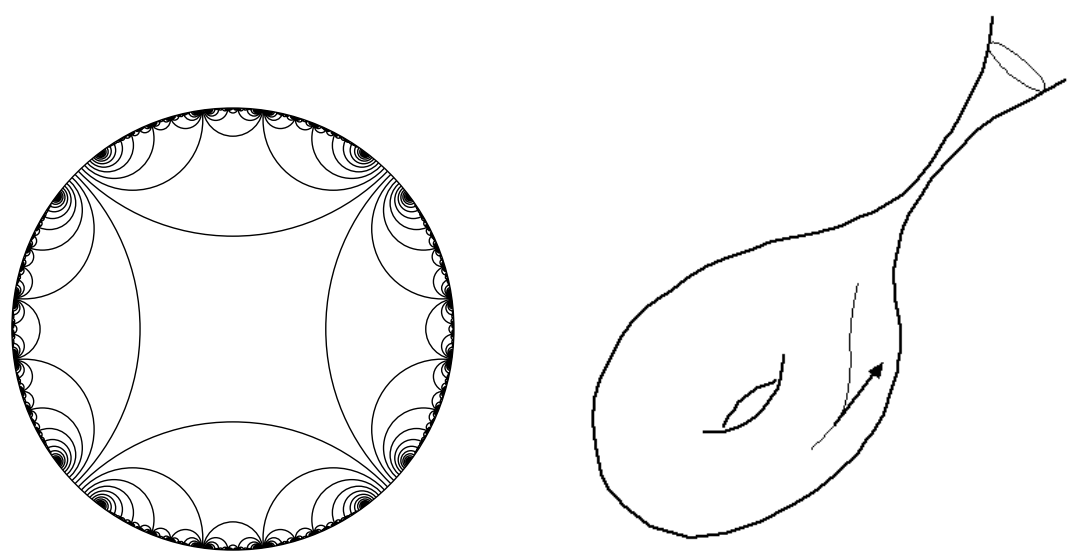

FigurE 3. After opening the cusp; gluing, we now have infinite area.

The flows $g_{t}$ and $h_{t}=h_{t}^{s}$ do not commute, but do satisfy the following commutation relation:

$$
h_{b} g_{a}=g_{a} h_{e^{-a} b} .
$$

In other words, the diagram in the left part of Fig. 2 commutes. One can prove this algebraically, or see it geometrically in the upper half plane, as illustrated in the right-hand part of the figure.

Remark 2.1. Because of the commutation relation, the pair (geodesic flow, horocycle flow) gives an action of the $(a x+b)$-group (the real affine group) on $T^{1}(M)$. This already hints that there might be a relation with fractal geometry, as fractal sets generally exhibit symmetries with respect to both dilation and translation.

Observation. The commutation relation tells us that $h_{t}=h_{t}^{s}$ is isomorphic to a speeded-up version of itself. An ergodic theorist immediately will recognise that this is very special, as the entropy of a sped-up transformation or flow multiplies by that factor, so in this case:

$$
\operatorname{entropy}\left(h_{t}\right)=e^{-a} \cdot \operatorname{entropy}\left(h_{t}\right) .
$$


There are, thus, only two possibilities for the entropy of the flow $h^{s}\left(\right.$ or $h^{u}$ ): 0 , or $\infty$ !

We have already seen an example of zero entropy (the finite area Riemann surface case); next we shall see a situation where infinite entropy occurs, and this example will lead us into the fractal realm.

\section{Brownian motion and stable processes}

Let $\Omega$ be the space of continuous functions from $\mathbb{R}$ to $\mathbb{R}$, with the topology of uniform convergence on compact sets. This makes $\Omega$ into a Polish space, that is, a complete separable metric space, which is ideal from the point of view of measure theory.

Let $\mu$ denote Wiener measure on $\Omega$, conditioned to be 0 at time 0 , and defined both for future and past times. Then the scaling property of Brownian motion says: for $B \in \Omega$ and $a>0$,

$$
B(a t) / \sqrt{a}
$$

is "distributed like" $B(t)$. What this probability language means to an analyst is: the transformation $B \mapsto \Delta_{a}(B)$ defined by

$$
\left(\Delta_{a}(B)\right)(t)=B(a t) / \sqrt{a}
$$

preserves Wiener measure. To an ergodic theorist this suggests the following: defining

$$
g_{t}=\Delta_{e^{-t}},
$$

the action $g_{t}: \Omega \rightarrow \Omega$ is a measure- preserving flow! Next question: what flow is it? Answer: up to measure theoretic isomorphism, it is (the) Bernoulli flow of infinite entropy. (See [Fis87], and see [Fis04] for related work.) Call this the scaling flow of Brownian motion; geometrically it dilates time and rescales space appropriately to give another Brownian path.

Next, consider the increment flow,

$$
\left(\left(h^{u}\right)_{a}(B)\right)(t)=B(a+t)-B(a) .
$$

This simply shifts the origin point $(0,0)$ along the graph of $B(t)$, and since the process $B(t)$ has stationary increments, again preserves the measure $\mu$.

Now comes the magical part: these two flows satisfy the same commutation relation as the geodesic and horocycle flows of our surface! But what is $\left(h^{u}\right)_{t}$, measure-theoretically? Answer: it is now infinite entropy. (One way to see this is to note that the increment flow is naturally isomorphic to the shift flow on white noise, which is an infinite entropy Bernoulli flow; the isomorphism is given by integration). So we have our "infinite entropy horocycle flow" example.

It is clear that the same commutation relation holds for any self-similar process with stationary increments; examples are the stable processes, see [FL02]; see [Zä91] for closely related work. 


\section{Brownian zero sets}

This study of Brownian motion gives us almost for free another example, which will make the link to fractal subsets of the line. As is well known, the zero set of a Brownian path, $Z_{B} \subseteq \mathbb{R}$, has Hausdorff dimension $1 / 2$, and for the gauge function $\phi=(2 t \log \log (1 / t))^{\frac{1}{2}}$, has positive locally finite Hausdorff measure $H_{\phi}$. Now define the map from $\Omega$ to itself by $B(t) \mapsto L_{B}(t)$ where $L_{B}(t)=H_{\phi}\left(Z_{B} \cap[0, t]\right)$, the total measure up to time $t$ (and similarly for $t<0$, but with negative sign). Write $\mu_{Z}$ for the image measure on path space $\Omega$; this is Paul Lévy's local time. (Note: to visualize the local time $L_{B}(t)$ of a Brownian path $B$, it helps to know - by a theorem of Lévy - that local time paths are exactly maximum paths $M_{\widehat{B}}(t)=$ $\sup \{B(s): s \in[0, t]\}$ for a different Brownian path $\widehat{B}$.) The flow $g_{t}$ preserves these correspondences, hence the scaling flow on $\left(\Omega, \mu_{Z}\right)$ is also a Bernoulli flow of infinite entropy (being a factor of the flow on $(\Omega, \mu)$ ) [BF92]. But what about the increment flow? Here things change: we slide into the gaps of the local time; the appropriate measure has become infinite. We shall return to examine the consequences of this in $\S 11$.

\section{The extended Cantor function (or Devil's Staircase)}

The graph of Brownian local time is a continuous, nondecreasing function with a dense set of flat spots, reminiscent of the Cantor function- which suggests to us that we study that non-random example in a similar way. In [Fis92] we see an extended version of the usual Cantor function as depicted, say, in Mandelbrot's book [Man]. Note the upper and lower envelopes of the form $c t^{d}$ for $d=\log 2 / \log 3$, the Hausdorff dimension of the Cantor set. Now this extended function $L(t)$, defined to be identically 0 for $t \leq 0$, satisfies

$$
L(3 t) / 2=L(3 t) / 3^{d}=L(t) ;
$$

this means that for the scaling flow $g_{t}$ of exponent $d, g_{t_{0}}(L)=L$, where $t_{0}=\log 3$; in other words, the scaling flow of this graph is a single periodic orbit!

Rescaling this path represents zooming down toward the point 0 in the Cantor set. But though the set $C$ is, as we all know, "the same everywhere", on closer inspection this isn't quite so true. What do we see, for instance, if we slide the graph of $f$ over to the point $1 / 4$ (which happens to be in $C$ ) by the increment flow, and then begin rescaling? In this case, the orbit is no longer periodic, but will converge asymptotically to a different periodic orbit, one with twice the previous length: $2 \log 3=\log 9$. But now differently from the point 0 , there is asymptotic scenery on both sides of the point $1 / 4$ [Fis92].

And this is still very special: what happens when a general point of $C$ replaces the number 1/4? Answer: after taking the forward orbit closure, we get a mixing ergodic flow! This will be naturally isomorphic to the scenery flow of the fractal set $C$, with which we began the article. So now it is time to give some precise definitions. 


\section{The scenery flow}

Let $\Omega=\Omega\left(\mathbb{R}^{n}\right)$ be the collection of all closed subsets of $\mathbb{R}^{n}$; topologise this by taking first the Hausdorff metric on closed subsets of the one-point compactification and then taking the relative topology on this subset $\Omega$. This topology (the Attouch-Wetts or geometric topology, see $\S 2$ of [BF96]) makes $\Omega$ compact; for an example consider $F_{n}=\{n\}$; this sequence of subsets of $\mathbb{R}$ converges in $\Omega(\mathbb{R})$ to the empty set.

Define the magnification flow $g_{t}$ on $\Omega$ by $A \mapsto e^{t} \cdot A$. Choose a closed subset $F \subseteq \mathbb{R}^{n}$, choose a point $z \in F$, and define $\Omega_{(F, z)}$ to be the omega-limit set of $(F-z)$, the set translated so as to place $z$ at the origin. Thus,

$$
\Omega_{F, z}=\bigcap_{T \geq 0} \operatorname{closure}\left\{g_{t}(F-z): t \geq T\right\} .
$$

The flow $\left(\Omega_{F, z} ; g_{t}\right)$ is the scenery flow of the set $F$ at the point $z$.

Example. Let $C$ be the Cantor set $C \subseteq[0,1]$; define $\widehat{C}=\cup_{k=0}^{\infty} 3^{k} C$, so $3 \cdot \widehat{C}=\widehat{C}$. Then

$$
g_{\log 3}(\widehat{C})=e^{\log 3} \cdot \widehat{C}=\widehat{C},
$$

so the scenery flow of $C$ at 0 is $\left\{e^{t} \widehat{C}: t \in[0, \log 3]\right\}$, and this is a single periodic orbit of length $\log 3$. The total Hausdorff measure $L(t)=H_{d}(\widehat{C} \cap[0, t])$ gives a nondecreasing continuous function, the extended Cantor function, and this produces a flow isomorphism to the scaling flow of the Cantor function, described before.

Definition 6.1. The scenery flow of the set $F$ is the flow of magnification by $e^{t}$ on $\Omega_{F}$, the union of the scenery flow spaces of each point $z \in F$ (so $\Omega_{F}=\cup \Omega_{F, z}$ ).

One can show [Fis92] that the scenery flow of the set $C$ is naturally isomorphic to the following special flow: the height $\log 3$ suspension of the natural extension of the map $3 x(\bmod 1)$ on $C$. As a first consequence, since this flow is recurrent, a.e. orbit is dense and so the landscape encountered while zooming down toward a general point passes through all possible limiting scenes; the orbit closure is the whole space. Moreover, the flow is ergodic, so, by the Birkhoff ergodic theorem, the scenes will be encountered with the right frequency. So in particular this proves that the order-two (average) density exists and is a.s. constant. See [BF92] for a slightly different proof.

But what else can one say about this flow? The base map is isomorphic to the Bernoulli shift $\sigma$ with weights $(1 / 2,1 / 2)$ on $\Sigma=\Pi_{-\infty}^{\infty}\{0,1\}$ via ternary expansion, and this map has entropy $\log 2$. Now we see something interesting. By Abramov's formula, flow entropy = (base entropy) $/$ (expected return time), so we conclude: entropy $\left(g_{t}\right)=\log 2 / \log 3=d=\operatorname{dim}(C)$ ! This gives us a formula for dimension:

The Hausdorff dimension of $C$ equals the entropy of the scenery flow.

This formula is not always valid: a counterexample is a Brownian zero set; the dimension is $1 / 2$ but the scenery flow is isomorphic to the scaling flow on local 
time, which, as we have seen, has infinite entropy. Nevertheless, we wonder: is it possible that this formula is valid elsewhere?

\section{The Fuchsian case}

In fact we shall find just such an example in Fuchsian limit sets. Recall our modified punctured torus, where we opened up the cusp into a trumpet. The limit set $\Lambda$ of the group $\Gamma$ is now a Cantor subset of the boundary (Fig. 3; all the open intervals which are unions of the closed interval where the fundamental domain meets the boundary are removed, leaving a topological Cantor set). So, what is its scenery flow? Well, first of all, the Cantor set is a subset of the circle, and zooming down toward this we see a tangent line: the infinitesimal scenery of a circle is a line. That's rather boring! But if we consider the scenery flow of the limit set, things become much more interesting. What we will see asymptotically is a collection of fractal subsets of the tangent space to the circle (the real line) which is invariant under dilation. But what are these sets? The answer is satisfyingly simple: they are the limit set $\Lambda$ moved to the boundary $\mathbb{R}$ of $\mathbb{H}$, the upper-half space model of the hyperbolic disk. Here we have to allow all possible correspondences via Möbius transformations from $\Delta$ to $\mathbb{H}$ such that a point of the limit set occurs both at 0 and $\infty$. Next, what can we say about the dynamics or ergodic theory of this scenery flow? We find:

Theorem 7.1. Let $\Delta$ be the unit disk with Poincaré metric, with boundary $\partial \Delta=S^{1}$. Let $\Gamma$ be a finitely generated Fuchsian group of second type (that is, the limit set $\Lambda$ is not all of the boundary). Then the scenery flow of $\Lambda$ is a finite-to-one factor of the geodesic flow of the surface $M=\Gamma \backslash \Delta$, and the limiting scenes are images of $\Lambda$ in $\mathbb{R}$ with respect to Möbius transformations from $\Delta$ to $\mathbb{H}$.

Proof. We construct the factor map directly. Let $v_{p}$ be a unit tangent vector based at the point $p \in \Delta$. Consider the geodesic tangent to $v_{p}$; this is a circle which meets $\partial \Delta$ orthogonally in two points $\eta, \xi$ in the past, future directions respectively. Since a complex Möbius transformation is determined uniquely by where it sends three points, there exists a unique Möbius transformation $\alpha=\alpha_{v_{p}}$ such that $\alpha$ takes $v_{p}$ to the unit vector $-i_{i}$ which points in the direction $-i$ at the location $i \in \mathbb{H}$, taking $p$ to $i, \eta, \xi$ to $\infty, 0$ and the geodesic to the imaginary axis.

More precisely, $\alpha(p)=i$ and $\alpha^{*}\left(v_{p}\right)=-i_{i}$, where $\alpha^{*}$ is the derivative of $\alpha$. Now define a map $\hat{\pi}: T^{1}(\Delta) \rightarrow \Omega=\{$ closed subsets of $\mathbb{R}\}$ by $v_{p} \mapsto \alpha_{v_{p}}(\Lambda)$.

Claim 1. For $\gamma \in \Gamma, \hat{\pi} \circ \gamma=\hat{\pi}$.

This holds since $\gamma(\Lambda)=\Lambda$.

Now let $\hat{g}_{t}$ be the geodesic flow on $T^{1}(\Delta)$.

Claim 2. $\hat{\pi}\left(\hat{g}_{t} v_{p}\right)=e^{t} \cdot \hat{\pi}\left(v_{p}\right)$.

The reason is that in $\mathbb{H}$, moving along the geodesic tangent to $-i_{i}$ (the imaginary axis) toward the point $0 \in \mathbb{H}$ is isomorphic by conjugacy to keeping the vector fixed at location $i$ and dilating $\mathbb{H}$ by the factor $e^{t}$. 
Claim 1 tells us that $\hat{\pi}$ induces a well-defined map $\pi: T^{1}(M) \rightarrow \Omega$ on the factor space $M=\Gamma \backslash \Delta$.

Claim 3. $\pi$ is finite-to-one.

Proof: Suppose $\hat{\pi}\left(v_{p}\right)=\hat{\pi}\left(w_{q}\right)$ for some other vector $w_{q}$ tangent to $\Delta$. Then $\left(\alpha_{w_{q}}\right)^{-1} \circ \alpha_{v_{p}}$ is a Möbius transformation of $\Delta$ which preserves the limit set $\Lambda$.

Let $\widetilde{\Gamma}$ be the subgroup of $\operatorname{Möb}(\Gamma)$ which has been extended from $\Gamma$ by adjoining all such elements. Then the limit set of $\widetilde{\Gamma}$ is also $\Lambda$. Now Margulis' Lemma implies that if $\Gamma_{1} \subseteq \Gamma_{2}$ are groups of hyperbolic isomorphisms of $\mathbb{H}^{n}$ and have the same limit set, then $\Gamma_{1}$ is of finite index in $\Gamma_{2}$. Hence in the factor space $T^{1}(M)$ there are at most finitely many such vectors $w_{q}$ for a given $v_{p}$.

We thank Bernie Maskit and Peter Waterman for their help with this part of the proof.

It remains to show:

Claim 4. The asymptotic limiting sceneries of the limit set in the circle $S^{1}$ are the images by the stereographic projections.

Proof: Choose a point $z$ in the limit set and place the circle so this point is at the origin, in the upper half space and tangent to the real axis. Zooming toward $z$ for time $t$ is equivalent to dilating this picture by the factor $e^{t}$; let us at the same time consider the downward-pointing unit vector at Euclidean height 1 in each picture. The Poincaré disks get larger and larger, approximating $\mathbb{H}$, and our tangent vector is moving via the geodesic flow. Now superimpose on this picture the stereographic projection determined by that vector, that is, by its image in the original Poincaré disk. The Möbius transformations converge to stereographic projections, proving the Claim.

We mention that the above proof extends immediately to limit sets of geometrically finite groups of isometries of hyperbolic $n$ - space $\mathbb{H}^{n}$. There is one main change: "geodesic flow" is replaced by "geodesic frame flow". Consider for instance $n=3$. Then $\Delta^{3}$ is the unit ball in $\mathbb{R}^{3}$ with the Poincaré metric; a frame is an orthonormal set of three vectors, one tangent to the geodesic flow, and the frame flow by definition simply moves this triple according to parallel transport. The choice of a frame defines a unique "stereographic projection" to the upper half-space model $\mathbb{H}^{3}$, sending the frame $f_{p}$ to the standard basis frame based at the point $(0,0,1) \in \mathbb{H}^{3} \subseteq \mathbb{R}^{3}$. The reason we need frames is that pictures of the scenery flow are different when rotated, and the frame flow will include this information.

\section{Ergodic theory and Sullivan's formula}

For the case of a Fuchsian group of second type, the geodesic flow of the surface $M=\Gamma \backslash \Delta$ at first glance is not nice from the point of view of ergodic theory: not only does $T^{1}(M)$ have infinite volume (this is not a priori a problem, as infinite measure ergodic theory can be brought in) but, much worse than that, a.e. vector $v_{p}$ is non-recurrent, i.e. eventually leaves any compact region. Sullivan's insight 
is that interesting dynamics can be recovered if we restrict attention precisely to those vectors which are recurrent for positive and negative times; these are exactly those vectors $v_{p}$ for which the endpoints $\eta, \xi$ at $-\infty,+\infty$ belong to the limit set $\Lambda$.

Next question: is there a natural measure to put on this recurrent set, replacing Riemannian volume? Sullivan's answer is a modification of Patterson's measure $\mu$ on $\Lambda$. (For nice cases, $\mu$ is the Hausdorff covering measure on $\Lambda$; for some other cases, as Sullivan showed, it is the packing measure.) Sullivan's measure on $T^{1}(M)$ is described as follows. It is defined first on $T^{1}(\Delta)$, in a $\Gamma$-equivariant way; hence it projects to the factor space $T^{1}(M)$. A unit tangent vector $v_{p}$ is parametrized by the two endpoints $\eta \neq \xi$, plus a real number (where it is along the geodesic). Hence $T^{1}(\Delta)$ can be parameterized by $\left(S^{1} \times S^{1}-\right.$ diagonal $) \times \mathbb{R}$. The recurrent set is represented by $(\Lambda \times \Lambda-$ diagonal $) \times \mathbb{R}$. The measure is equivalent (shares the same sets of measure zero) with $(\mu \times \mu) \times$ Lebesgue measure; the Radon-Nikodym derivative with respect to this product measure is $1 /|\eta-\xi|^{2 d}$, with distance measured in the Euclidean metric on the disk and $d$ the Hausdorff dimension of $\Lambda$.

This guarantees that the resulting measure (known as the Patterson-Sullivan measure ) is $\Gamma$-equivariant and invariant for the geodesic flow. Sullivan then proves the following theorem (we state the $n$-dimensional version); some background references are [Pat76],[Pat87], [Sul84], [Sul70]:

Theorem 8.1. Let $\Gamma$ be a geometrically finite subgroup of $M \ddot{b} b\left(\Delta^{n}\right)$. Then the geodesic flow is ergodic for the Patterson-Sullivan measure $\hat{\mu}$, and $\hat{\mu}$ is the unique measure of maximal entropy, with entropy equal to the Hausdorff $\operatorname{dimension} \operatorname{dim}(\Lambda)=$ $d$.

We can hence conclude:

Corollary 8.2. The topological entropy of the scenery flow of the limit set of a geometrically finite Kleinian group is equal to the Hausdorff dimension of that set.

Proof. The scenery flow is a finite index factor of the frame flow. The entropy of the frame flow equals that of the geodesic flow, since it is an isometric extension. Since it is a finite-to-one factor, entropy is preserved.

\section{The scenery flow of a Julia set and hyperbolic Cantor set}

The definition of the scenery flow (as an omega-limit set of the magnification flow acting on closed subsets of $\mathbb{R}^{n}$ ) makes sense for general fractal sets; sometimes we can identify this flow, constructing it concretely. We have already discussed the example of the middle-1/3 Cantor set; this is a linear Cantor set, and many other such linear flows (such as those generated by linear conformal IFS's with the open set condition) can be studied by a straightforward modification of this. The case of Kleinian limit sets was also easy, as this is also a "linear" case; the maps are linear 
fractional transformations, and, as we have seen above, the limiting sceneries are just given by stereographic projection.

We describe next the case of hyperbolic Julia sets; conformal mixing repellors and hyperbolic $\mathbb{C}^{1+\alpha}$ Cantor sets are dealt with in a similar way.

There is an analogy to the Kleinian case. There we had a homomorphism $\pi$ from the hyperbolic manifold $T^{1}(M)=T^{1}(\Gamma \backslash \Delta)$ to the space $\Omega$ of scenes, conjugating the geodesic frame flow to the magnification flow on sets.

Here we construct a "model scenery flow" which will play the role of $T^{1}(M)$.

Let $f: \mathbb{C} \rightarrow \mathbb{C}$ be a rational map which is hyperbolic on its Julia set $\mathcal{J}$. Write $D f$ for its derivative, and $f_{*}$ for the action of the derivative on the unit tangent bundle of $\mathcal{J}$ (by which we mean the unit tangent bundle of $\mathbb{C}$, restricted to $\mathcal{J})$. Let $\tilde{f}: \widehat{\mathcal{J}} \rightarrow \widehat{\mathcal{J}}$ denote the natural extension of this map. The space $\widehat{\mathcal{J}}$ is an inverse limit and can be identified with the space of sequences $\underline{\hat{z}}=\left(\ldots \hat{z}_{-1} \hat{z}_{0} \hat{z}_{1} \ldots\right)$ where $\hat{z}_{i}=\left(z_{i}, \theta_{i}\right) \in \mathbb{C} \times[0,2 \pi]$ represents a unit tangent vector and the sequence satisfies $\hat{z}_{i+1}=f_{*}\left(\hat{z}_{i}\right)$. Write $\widehat{\Omega}$ for this collection of sequences, with shift map $\hat{\sigma}$; let $\widetilde{\Omega}$ denote the suspension flow over $(\widehat{\Omega}, \hat{\sigma})$ with height (= return time) $\log \left|D f\left(z_{0}\right)\right|$. This is our model flow.

The flow homomorphism $\pi$ from $\widetilde{\Omega}$ to $\Omega$ is defined as follows: on the base, $\pi(\underline{\hat{z}})=L_{\underline{\hat{z}}}=\lim _{n \rightarrow \infty}\left(D f^{n}\left(z_{-n}\right) \cdot\left(\mathcal{J}-z_{-n}\right)\right)$. The limit is in the Hausdorff metric on closed subsets of $\widehat{\mathbb{C}} \equiv \mathbb{C} \cap\{\infty\}$; thus the Julia set has been centered at the $n^{\text {th }}$ preimage $z_{-n}$ and expanded and rotated by the derivative map. That this always converges is a consequence of bounded distortion, see [BFU]. We have:

Theorem 9.1. ([BFU], [FU]). For the hyperbolic Julia set of a rational map, there is, up to rotation, a unique measure of maximal entropy for the model scenery flow. Its entropy satisfies the formula "model flow entropy equals $\operatorname{dim}(\mathcal{J})$ ". When we include rotations, then for all but a few exceptional cases, there is a unique measure of maximal entropy and the model scenery flow is ergodic with respect to this measure.

Remark on proof. We refer the reader to [BFU], but mention two interesting points. First, ergodicity for the model flow implies a rotational symmetry for the scenery flow; that is, when zooming down toward a.e. point we not only eventually see all possible scenes, but they occur at all angles (and with the expected frequency). This question can, by a lemma of Furstenberg [Fur61] see [BFU] be formulated in terms of the non-existence of a certain circle-valued cocycle or equivalently as a problem about the non-existence of Hausdorff measure invariant line fields (or cross fields); so, the hard work in proving ergodicity has been transferred to that setting: see [FU] and [May].

Second, we sketch here the proof of the entropy formula because it is so instructive and works out so nicely. Bowen's formula for dimension [Bo79] reads: there exists a unique $d$ such that $P\left(-\log |D f|^{d}\right)=0$; this is $\operatorname{dim}(\mathcal{J})$. Here $P(\phi)$ is the pressure of a function $\phi$, which can be defined as $P(\phi)=\sup \left(h(\mu)+\int \phi \mathrm{d} \mu\right)$ where the supremum is taken over invariant probability measures and $h(\mu)$ is the 
entropy of the transformation $(\mathcal{J}, f, \mu)$. We know from the theory of Sinai-RuelleBowen that there exists a unique invariant measure $\mu$ such that the sup is attained. So we have for this invariant measure $\mu$ :

hence

$$
0=h(\mu)+\int-\log |D f|^{d} \mathrm{~d} \mu
$$

$$
h(\mu)=\int \log |D f|^{d} \mathrm{~d} \mu=d \int \log |D f| \mathrm{d} \mu
$$

and so

$$
\frac{h(\mu)}{\int \log |D f| \mathrm{d} \mu}=d .
$$

Now the formula on the left is (base entropy)/(expected return time), hence (by Abramov's formula) equals the special flow entropy.

The scenery flow is, a priori, a magnification flow defined on sets; however, it is natural to carry along more information, given (for the previous examples) by a labelling inherited from the Kleinian group or map $f$ respectively. This information is provided by the model flows; we call this the labelled or marked scenery flow. We conclude with a dimension formula which unites Sullivan's formula for Kleinian limit sets, with Bowen's for Julia sets and "cookie cutter" Cantor sets:

Theorem 9.2. For geometrically finite Kleinian groups in dimension n, hyperbolic rational maps, conformal mixing repellors, and hyperbolic $\mathbb{C}^{1+\alpha}$ Cantor sets, we have the formula:

The topological entropy of the marked scenery flow of the limit set is equal to the Hausdorff dimension of the limit set.

\section{Doubling maps and the Riemann surface lamination}

The simplest example of a hyperbolic Julia set is for the map $f(z)=z^{2}+c$ with $c=0$; then $\mathcal{J}$ is the circle $S^{1}$ and $f$ restricted to $\mathcal{J}$ is the usual doubling map of $S^{1}$, isomorphic to $x \mapsto 2 x(\bmod 1)$ on the unit interval. In this case, the natural extension of $f$ is the hyperbolic map on the solenoid $\hat{f}: \widehat{S} \rightarrow \widehat{S}$, which in turn is an a.s. one-to-one factor of the Bernoulli shift $\sigma: \Sigma \rightarrow \Sigma$ for $\Sigma \equiv \Pi_{-\infty}^{\infty}$. Here $|D f|=2$ so the model flow is the special flow of height $\log 2$ over the solenoid. The base entropy is $\log 2$, so the flow entropy is (by Abramov) $\log 2 / \log 2=1$, which is, indeed, the Hausdorff dimension of the Julia set $S^{1}$. In this case the model flow space is identical to Sullivan's Riemann surface lamination [Sul92], [Sul91]; we thank especially D. Sullivan and J. Kahn for conversations on this point. See also Chapter VI of [dMvS] regarding these papers of Sullivan.

This suggests that the model flow for a general hyperbolic Julia set is, on the one hand, a generalization of the Riemann surface lamination and on the other, an analogue for rational maps of the recurrent part of the frame bundle 
of the hyperbolic $n$-manifold $\Gamma \backslash \Delta^{n}$. Minsky and Lyubich, building partly on our construction of sceneries, and extending that to general rational maps, showed the following remarkable result: that a rigidity theorem of Thurston for rational maps can be proved analogously to Mostow's rigidity theorem for Kleinian groups, replacing the 3-manifold by the "hyperbolic 3-manifold lamination". See [ML], and $[\mathrm{Lyu}]$ for related work. All of this, also [BFU] and [FU], fits the philosophy of the Sullivan-Thurston "dictionary" between Kleinian groups and rational maps.

The Riemann surface lamination is a double suspension: the solenoid is a suspension of the adic transformation (odometer), giving a flow $\left(h^{u}\right)_{t}$, and the scenery flow space is a suspension flow $g_{t}$ over that (now suspending the hyperbolic map). This space carries two flows: the flow which spins around the solenoid direction, which is just the lift of the rotation flow on the circle, and which preserves each level, and the vertical flow. This pair of flows $h^{u}, g$ satisfy the same commutation relation as before-which is, indeed, exactly what should happen, since the solenoid leaves at a given height are the unstable leaves of the vertical flow.

\section{The horocycle flow: infinite measures, return times and average density}

Let us consider the case of a Fuchsian group of first type (the limit set is the circle); here the appropriate measure for the geodesic flow and for the horocycle flows $h^{u}, h^{s}$ is the same: Riemannian volume on $T^{1}(M)$. For a group of second type, the situation is radically different: our recurrent measure is Patterson-Sullivan measure $\hat{\mu}$, but this is no longer invariant for $h^{u}$ or $h^{s}$. The reason is that any given horocycle tangent to the limit set $\Lambda$ meets the recurrent set in a fractal subset of the horocycle. The natural measure, therefore, is a modification of this: it is now equivalent to (Lebesgue measure $\times \mu$ ) $\times$ Lebesgue measure. That is, e.g. for $h^{s}$, the boundary point $\xi$ at $+\infty$ of a vector $v_{p}$ in the support of this measure is required to be in the limit set, while the infinite past boundary point $\eta$ is free to wander along the real line; these points have conditional measure given by Patterson measure $\mu$ and by Lebesgue measure on $\mathbb{R}$ respectively.

In nice cases, this measure (which we call Kenny measure), is the unique invariant Radon measure up to multiplication by a constant (since it is infinite there is always this choice of normalization). See [Ken] for the general no-cusp case, [Bur] for the no-cusp case with dimension $>1 / 2$. With M. Burger we have a proof different from Kenny's which includes the case with cusps allowed (where we rule out atomic measures and measures supported on horocycles around the cusps in the statement of unique ergodicity); manuscript in preparation.

It is natural to wonder what happens for other fractal sets. For instance for hyperbolic $\mathbb{C}^{1+\alpha}$ Cantor sets, one can also prove infinite-measure unique ergodicity, using techniques of [BM], combined with [BF96] and [BF97], see also [Fis03a].

The philosophy suggested by these examples is:

For some infinite measure preserving transformations,

the returns to a finite measure subset are a fractal-like subset of times. 
This idea of a "fractal-like" subset of the integers can be made precise by use of the scaling flow (as $t \rightarrow-\infty$ for the way we have defined that flow here). The notion of average (or order-two) density extends to integer fractal sets, playing the role of a finitely additive Hausdorff measure, see [BF92]. There is another interpretation: since the average density can be thought of as a parameter which measures the lacunarity of a fractal set, we have here an analogue of Mandelbrot's concept [Man], for subsets of the integers. The integer average density leads to a new type of ergodic theorem for certain infinite measure transformations, given by normalization by the "dimension" followed by a log average [Fis92]. Then the average density reappears in a different guise: the limiting value of the time average is the expected value of the observable times the average density of the fractal integer set. The fractal point of view leads to two new isomorphism invariants for infinite measure-preserving transformations: the dimension of return times, and the average density (providing the defining limits exist). See [Fis92] and [Fis03b].

Further insight is given by examples coming from probability theory. Certain countable state Markov chains [Fel] exhibit this type of behavior. This led us to an extension of a beautiful but little-known result of Chung and Erdös [CE], see $[\mathrm{ADF}]$.

Now since infinite measure-preserving transformations can have a geometrical significance, related to fractal sets, one might wonder whether there might be examples of a transition from finite to infinite measure, based on this point of view; that might be regarded as analogous to a change of phase in physics.

Just such a phenomenon occurs for certain maps of the interval with an indifferent (or neutral or parabolic) fixed point. Here there is a 1- parameter family of maps (see [FL01], [FL02], [FL04a], [FL04b]), related to the Markov chain examples just discussed and also to the Manneville-Pomeau maps (see also [Lop]) as well as to the interesting counterexamples of Hofbauer [Hof], which exhibits three "phases" of behavior, as the parameter $\alpha$ ranges from 0 to $\infty$.

We consider the distribution of returns to the right half of the interval. For $\alpha \in(2, \infty)$, the mean and variance are finite; for $\alpha \in(1,2)$, variance is infinite but mean is finite, while for $\alpha \in(0,1)$, both are infinite. For $(1,2]$ and $(2, \infty)$ the unique absolutely continuous invariant measure for the map is finite; for $(0,1]$ it is infinite. The asymptotic return-time behavior for $[2, \infty)$ is Gaussian; for $(1,2]$ it is stable, passing through all the completely asymmetric stable laws; for $(0,1)$ it continues on, through all the Mittag-Leffler processes.

This last region (infinite measure) is the realm of fractal-like return times. The Mittag-Leffler paths are similar to Cantor functions. The increment flow along these processes has infinite measure (for background see [Aar], [Zwe]), and is a horocycle flow for the corresponding scaling flow (which is the "geodesic flow"). For all parameters $\alpha \neq 1$ the scaling flow on paths is infinite entropy Bernoulli (the case $\alpha=1$ is handled in a special way as it has an extra drift parameter). This example completes a circle of ideas begun in [Fis92], [ADF]. 


\section{Spaces of tilings}

Fractal sets are but one of the geometric forms with an interesting small-scale structure. Other examples are tilings of $\mathbb{R}^{n}$ which have some sort of self-similar nesting character.

The simplest example is the binary tiling of the unit interval; at level $n \geq 0$, there are $2^{n+1}$ tiles given by the intervals of the form $\left[k \cdot 2^{-(n+1)},(k+1) \cdot 2^{-(n+1)}\right]$. The tiling structure is generated by the dynamics of the map $f: x \mapsto 2 x(\bmod 1)$ and can be thought of as a "cookie cutter Cantor set without the gaps". Spaces of nested tilings of $\mathbb{R}^{m}$ are topologized in a natural way related to the AttouchWetts topology discussed above: the hierarchy of tilings is indexed by $\mathbb{Z}$; two nested tilings are close if on a large ball in $\mathbb{R}^{m}$, the boundaries are $\varepsilon$-close in the Hausdorff metric, for each index $l$ between $-k$ and $k$. The scenery flow for this space of tilings is modelled by the height $\log 2$ suspension flow over the natural extension of $f$, discussed before, i.e. by Sullivan's Riemann surface lamination.

Now the binary tilings are the joins of pullbacks of the standard Markov partition $\mathcal{P}=\left\{P_{0}, P_{1}\right\}=\{[0,1 / 2],[1 / 2,1]\}$ for this map. This suggests that Markov partitions give interesting candidates for a scenery flow. A next example to consider is that of an Anosov toral diffeomorphism, such as $\left[\begin{array}{ll}2 & 1 \\ 1 & 1\end{array}\right]$. Here we will have two scenery flows, one for the stable and one for the unstable foliation. The resulting model scenery flow (for the unstable case) is the height $\log \left|D f^{u}\right|$ suspension of the Anosov diffeomorphism; the horocycle flows are the translations of the tilings, and this is modelled by the unstable flow of the suspension.

Many fractal tilings associated to "complex number systems" can be treated in a similar way, with more complicated Markov partitions. A theorem of Bowen [Bo78] says that a Markov partition for an $n$-toral automorphism for $n \geq 3$, or toral endomorphism for $n \geq 2$, cannot have a smooth boundary. Examples with fractal Markov partition boundaries were studied by Bedford [Bed], see also [Dek], [Pra], $[\mathrm{KV}],[\mathrm{Ma} 02]$. The scenery flow of the hierarchy of nested tilings generated by such Markov partition is constructed in a similar way as for the doubling map of the circle, see $\S 10$.

Another class of interesting one-dimensional tilings are those given by the renormalization hierarchy of an interval exchange transformation. See [AF03] for a development of the basic theory and an in-depth study of the simplest example (exchanges of two intervals), and [AF01], [Fis03b] for more information.

If all exchanges of $k$ intervals are considered as parameter space, the construction places parameter space and dynamical space (the interval exchanges themselves) in a single unified picture. Following this example, we can return to the geodesic and horocycle flows of a Riemann surface, and reinterpret them in this new light. The commutation relation now says: The horocycle flow is a fixed point of renormalization, as the geodesic flow renormalizes the horocycle flow to itself. This may make us think of the scenery flow of the attracting Cantor set $C$ of the Coullet-Tresser-Feigenbaum map $f$, also a fixed point for a renormalization 
operator. There is a second map $g$, conjugate to the shift $\sigma$, which has an analytic extension, and for which $C$ is a repellor, see [Fal85], [Ran], [Sul87]. Using this hyperbolic map $g$ we construct its scenery as in $\S 9$. The maps $g$ and $f$ satisfy the commutation relation $g \circ f^{2}=f \circ g$, a discrete-time version of that for the geodesic and horocycle (dilation and translation) scenery flows.

\section{Nonlinear doubling maps and smooth classification}

Now we return to the basic example of the doubling map on the circle, but with a twist which makes things even more interesting: we take a nonlinear version, given by a degree 2 hyperbolic $\mathbb{C}^{1+\alpha}$ map $f: S^{1} \rightarrow S^{1}$. Using the same machinery discussed above for Julia sets, and similar also to the cookie cutter Cantor set case of [BF97], one constructs the scenery flow for the space of tilings. This is a complete invariant of smooth conjugacy. A closely related invariant is Sullivan's scaling function [Sul87]. We construct a solenoid much as before, but with relative lengths of subtiles now given by this scaling function. As Sullivan noted [Sul92] there is a correspondence between conformal structures on the Riemann surface lamination and scaling functions. See Theorem VI.6.1 of [dMvS] for a proof in the analytic case.

The scenery flow picture helps to make the conformal structure more easily understood: for it is just the natural conformal structure on the height $\log |D f|$ suspension flow over the solenoid. It turns out that a smooth equivalence class of hyperbolic $C^{1+\alpha}$ maps is determined by the topological data (degree) plus any one of these: a Gibbs measure class, a Hölder scaling function, a scenery flow with Hölder return time, and a conformal structure on the Riemann surface lamination.

Here is one way of viewing all this. Given a hyperbolic doubling map, Cantor set or Julia set, the construction of the scenery is a sort of linearization procedure - and the space of scenes is a tangent object, the analogue of a tangent space. As such, it is acted on (linearly) by the derivative of the map. This gives the return map to a cross-section of the scenery flow. The flow itself is simply dilation, and is linear as well; the nonlinearity of the original map has been coded into the flow space, by means of the identifications made when defining that space; and that information is, in turn, remembered by the conformal structure of the Riemann surface lamination.

\section{Further directions of research; Acknowledgements}

Some classes of examples not mentioned so far are self-affine fractals (as for example the graphs $(t, B(t))$ of Brownian motion paths, see U. Zähle [Zä91]), spacefilling curves (on which the author is currently working with P. Arnoux), and spatially inhomogeneous fractals such as the "theater curtain" pictures in [Man], which interpolate middle- interval Cantor sets from the whole unit interval to the empty set, see also p. 107 of [Fal97] and [PZ]. The holy grail of non-homogeneous examples must be the Mandelbrot set boundary $M$. As Tan Lei showed [Tan], at certain points of $M$ the scenery flow can be precisely analysed: for a Misiurewicz 
point $c$, the scenery flow of $M$ at $c \in M$ is that of the Julia set $\mathcal{J}_{c}$ at $c$ for the map $z \mapsto z^{2}+c$. However what happens at this set of points, even though it is a countable dense subset of $M$, says next to nothing about the nearby points. The limiting scenery there is anybody's guess (though see $[\mathrm{McM}]$ regarding "deep points") and seems to be a deep and difficult problem.

Lastly we mention two closely related but quite complementary approaches to the study of small-scale structure of fractal sets, each of which is much like what we have called the scenery. Furstenberg's microsets were motivated especially by the study of intersections, sums and products of arithmetically derived fractal sets see [Fur70]. Preiss' tangent measures were introduced to study geometric measures at small scales [Pre], [Mat]. For certain self-similar measures, the tangent measures at almost every point form the same tangent measure distribution [Gra]. Mörters and Preiss $[\mathrm{MP}]$ have shown that tangent measure distributions are so-called Palm measures, and so have connected the geometric study of measures to an important series of papers by Ulrich Zähle [Zä88], [Zä90], [Zä91] who studied self-similarity of random measures. See Patzschke and M. Zähle [PZ], Moerters (who generalized the average density introduced in [BF92]) [Mö97], [Mö98], [KM], Falconer and Xiao [Fal97], [FX], [Xia] for some related developments.

Perhaps the future will see a general theory which integrates all these approaches. My own sense is that our understanding is still at a very beginning stage, and that there is a world of exciting exploring yet to be done at the small scales of these remarkable landscapes.

I wish especially to thank coworkers on these and related projects: Jon Aaronson, Pierre Arnoux, Tim Bedford, Marc Burger, Herold Dehling, Manfred Denker, Sebastien Ferenczi, Pascal Hubert, Artur Lopes, Jerome Los, Yair Minsky, Luis Fernando Carvalho da Rocha and Mariusz Urbański. Figures 1 and 3 were made using McMullen's lim program; Tony Phillips helped with the other graphics. There are many more people who should be acknowledged here, see [Fis03b]. I wish to add that without the encouragement and participation of these friends, teachers, and colleagues, most of this would never have been worked out and in any case the process would not have been nearly so much fun.

\section{References}

[Aar] J. Aaronson. An Introduction to Infinite Ergodic Theory. Math. Surveys and Monographs. AMS, 1997.

[ADF] J. Aaronson, M. Denker, and A. M. Fisher. Second order ergodic theorems for ergodic transformations of infinite measure spaces. Proc. Amer. Math. Soc. 114(1):115-128, 1992.

[AF01] P. Arnoux and A.M. Fisher. The scenery flow for geometric structures on the torus: the linear setting. Chinese Ann. of Math., 4:427-470, 2001.

[AF03] P. Arnoux and A. M. Fisher. Anosov families, renormalization and nonstationary subshifts. Technical report, LMD, Univ. de Marseilles, 2003.

[Bed] T. Bedford. Generating special Markov partitions for hyperbolic toral automorphisms using fractals. Ergod. Th. Dynam. Sys., 6:325-333, 1986. 
[BF92] T. Bedford and A.M. Fisher. Analogues of the Lebesgue density theorem for fractal sets of reals and integers. Proc. London Math. Soc., 64:95-124, 1992.

[BF96] T. Bedford and A.M. Fisher. On the magnification of Cantor sets and their limit models. Monatsh. Math., 121:11-40, 1996.

[BF97] T. Bedford and A.M. Fisher. Ratio geometry, rigidity and the scenery process for hyperbolic Cantor sets. Erg. Th. Dyn. Sys., 17:531-564, 1997.

[BFU] T. Bedford, A.M. Fisher, and M. Urbański. The scenery flow for hyperbolic Julia sets. Proc. London Math. Soc., 3(85):467-492, 2002.

[BM] R. Bowen and B. Marcus. Unique ergodicity for horocycle foliations. Israel Jour. Math., 26(1):43-67, 1977.

[Bo78] R. Bowen. Markov partitions are not smooth. Proceedings of the American Mathematical Society, 71:130-132, 1978.

[Bo79] R. Bowen. Hausdorff dimension of quasi-circles. Publications Mathématiques (Institut des Hautes Etudes Scientifiques, Paris), 50:11-25, 1979.

[Bur] M. Burger. Horocycle flow on geometrically finite surfaces. Duke Math. J., 61(3):779-803, 1990.

[CE] K.L. Chung and P. Erdös. Probability limit theorems assuming only the first moment I. Memoirs Amer. Math. Soc., 6, 1951.

[Dek] M. Dekking. Recurrent sets. Adv. in Math., 44:78-104, 1982.

[dMvS] Welington de Melo and Sebastien van Strien. One-Dimensional Dynamics. Springer, 1993.

[Fal85] K.J. Falconer. The Geometry of Fractal Sets. Cambridge U. Press, New York/Cambridge, 1985.

[Fal97] K. Falconer. Techniques in Fractal Geometry. John Wiley and Sons, 1997.

[Fel] W. Feller. Fluctuation theory of recurrent events. Trans. AMS, 67:98-119, 1949.

[Fis87] A.M. Fisher. Convex-invariant means and a pathwise central limit theorem. $A d v$. Math., 63:213-246, 1987.

[Fis92] A.M. Fisher. Integer Cantor sets and an order-two ergodic theorem. Ergod. Th. Dynam. Sys., 13:45-64, 1992.

[Fis03a] A.M. Fisher. Notes on Dynamics: the ergodic theory of adic transformations, 2003.

[Fis03b] A.M. Fisher. Small-scale Structure and Randomness: the Scenery Flow in Dynamics, Geometry and Probability. http://ime.usp.br/ afisher, March 2003. thesis for livre docencia, Univ. of São Paulo.

[Fis04] A.M. Fisher. A Pathwise Central Limit Theorem for random walks. Adv. Prob. (to appear), 2004.

[FL01] A.M. Fisher and A. Lopes. Exact bounds for the polynomial decay of correlation, 1 /f noise and the CLT for the equilibrium state of a non-Hölder potential. Nonlinearity, 14:1071-1104, 2001.

[FL02] A.M. Fisher and A. Lopes. Invariance principles in log density and convergence to stable flows. Technical report, USP, 2002.

[FL04a] A.M. Fisher and A. Lopes. Invariance principles in log density for renewal processes. Technical report, USP, 2004. 
[FL04b] A.M. Fisher and A. Lopes. Self-similar return sets and stable laws for some maps with an indifferent fixed point. Technical report, USP, 2004.

[FU] A.M. Fisher and M. Urbański. On invariant line fields. Bulletin London Math. Soc., 32:555-570, 2000.

[Fur61] H. Furstenberg. Strict ergodicity and transformation of the torus. Amer. J. Math., 83:573-601, 1961.

[Fur70] H. Furstenberg. Intersections of Cantor sets and transversality of semigroups. In R. C. Gunning, editor, Problems in Analysis, a symposium in honor of $S$. Bochner. Princeton Univ. Press, 1970.

[FX] K.J. Falconer and Yimin Xiao. Average densities of the image and zero set of stable processes. Stochastic Processes Appl., 55:271-283, 1995.

[Gra] S. Graf. On Bandt's tangential distribution for self-similar measures. Monatsh. Math., 120:223-246, 1995.

[Hof] F. Hofbauer. Examples for the non-uniqueness of the equilibrium states. Trans. Amer. Math. Soc., 228:133-141, 1977.

[Ken] P. Kenny. Ergodic measures for a class of horocycle flows. PhD thesis, McGill University, 1983.

[KM] D. Krieg and P. Mörters. Tangent measure distributions of hyperbolic Cantor sets. Monatshefte Math., 312:53-93, 1998.

[KV] R. Kenyon and A. Vershik. Arithmetic construction of sofic partitions of hyperbolic toral automorphisms. Erg. Th. Dyn. Syst., 18:357-372, 1998.

[Lop] A. Lopes. The Zeta function, non-differentiabilty of pressure and the critical exponent of transition. Adv. in Math., 101(2):133-165, 1993.

[Lyu] M. Lyubich. Laminations and holomorphic dynamics. Technical report, Lecture Notes of the mini-course given at the Conference "New Directions in Dynamical Systems in Kyoto, August, 2002.

[Man] B. Mandelbrot. The Fractal Geometry of Nature. W. H. Freeman, 1982.

[Ma02] A. Manning. A Markov partition that reflects the geometry of a hyperbolic toral automorphism. Trans. Amer. Math. Soc., to appear.

[Mat] P. Mattila. Geometry of Sets amd Measures in Euclidean Spaces, volume 44. Cambridge Advanced Studies in Mathematics, 1995.

[May] V. Mayer. Comparing measures and invariant line fields. Ergod. Th. Dynam. Sys., 22:555-570, 2002.

[McM] C.T. McMullen. Renormalization and 3-manifolds which fiber over the circle, volume 142 of Studies in Math. Princeton Univ. Press, Princeton, New Jersey, 1996.

[ML] Y. Minsky and M. Lyubich. Laminations in holomorphic dynamics. J. Diff. Geometry, 47:17-94, 1997.

[Mö97] P. Mörters. Average densities, tangent measures and rectifiability. Periodica Mathematica Hungarica, 37:313-324, 1997.

[Mö98] P. Mörters. Average densities and linear rectifiability of measures. Mathematika, 37:65-79, 1998.

[MP] P. Mörters and D. Preiss. Tangent measure distributions of fractal measures. Math. Annalen, 126:313-328, 1998. 
[Pat76] S. J. Patterson. The limit set of a Fuchsian group. Acta Math, 136:241-273, 1976.

[Pat87] S. J. Patterson. Measures on limit sets of Kleinian groups. In D. B. A. Epstein, editor, Analytical and Geometric Aspects of Hyperbolic Space, volume 111 of LMS Lecture Notes Series, pages 281-323. Cambridge Univ. Press, 1987.

[Pra] B. Praggastis. Numeration systems and Markov partitions from self similar tilings. Trans. Amer. Math. Soc., 351:3315-3349, 1999.

[Pre] D. Preiss. Geometry of measures in $\mathbb{R}^{n}$ : Distribution, rectifiability, and densities. Annals Math., 125:537-643, 1987.

[PZ] N. Patzschke and M. Zähle. Fractional differentiation in the self-affine case III: The density of the Cantor set. Proc. AMS, 117:132-144, 1993.

[Ran] D.A. Rand. Global phase space universality, smooth conjugacies and renormalization: I. the $\mathcal{C}^{1+\alpha}$ case. Nonlinearity, 1:181-202, 1988.

[Sul70] D.P. Sullivan. The density at infinity of a discrete group of hyperbolic motions. Publ. Matématiques, 50:171-202, 1970.

[Sul84] D.P. Sullivan. Entropy, Hausdorff measures old and new, and limit sets of geometrically finite Kleinian groups. Acta Math., 153:259-277, 1984.

[Sul87] D.P. Sullivan. Differentiable structures on fractal-like sets, determined by intrinsic scaling functions on dual Cantor sets. AMS Proc. Symp. Pure Math., 48:15-23, 1987.

[Sul91] D.P. Sullivan. Bounds, quadratic differentials, and renormalization conjectures. AMS Centennial Publications, 2, 1991.

[Sul92] D.P. Sullivan. Linking the universalities of Milnor-Thurston, Feigenbaum and Ahlfors-Bers. In Topological Methods in Modern Mathematics, pages 543-564. Publish or Perish, 1992.

[Tan] Lei Tan. Similarity between the Mandelbrot set and Julia sets. Comm. Math. Phys., 134:587-617, 1990.

[Xia] Yimin Xiao. Random fractals and Markov processes. In Proceedings of the Conference on Fractal Geometry and Applications: A Jubilee of Benoit Mandelbrot, 2003. (to appear).

[Zä88] U. Zähle. Self-similar random measures I: Notion, carrying Hausdorff dimension, and hyperbolic distribution. Prob. Theor. Rel. Fields, 80:79-100, 1988.

[Zä90] U. Zähle. Self-similar random measures II: a generalization to self-affine measures. Math. Nachr., 146:85-98, 1990.

[Zä91] U. Zähle. Self-similar random measures III: Self-similar random processes. Math. Nachr., 151:121-148, 1991.

[Zwe] R. Zweimueller. Probabilistic properties of dynamical systems with infinite invariant measures. Diplomarbeit, Univ. Salzburg 1995.

Albert M. Fisher

Dept Mat IME-USP, Caixa Postal 66281, CEP 05315-970 São Paulo, Brazil

URL: http://ime.usp.br/ afisher

e-mail: afisher@ime.usp.br 